\title{
Understanding multiple sclerosis by examination of theories in medical literature
}

\begin{abstract}
Multiple sclerosis (MS) is a neurodegenerative disease which causes inflammation and demyelination in the central nervous system. The demyelination is caused from mediated cell death, apoptosis and/or necrosis, of oligodendrocytes. Although MS can be diagnosed, very little is understood about its pathogenesis. Many researchers have focused on the immune system itself in attempt to understand the pathogenesis of MS. The idea that demyelination primarily resulting from a $\mathrm{T}$ cell-mediated immune response to various myelin antigens is widely accepted; however, there are other factors to consider. In order to understand the primary cause(s) of MS which are not fully understood in science research currently, it is important to examine the effects of glutamate excitotoxicity and the environmental factors which could cause it. is opinion paper seeks to outline theories regarding the pathogenesis of MS in attempt to understand this complicated neurodegenerative disease.
\end{abstract}

Keywords: multiple sclerosis, oligodendrocytes, glutamate, excitotoxicity, autoimmune, neurodegenerative, disease
Volume 3 Issue 5 - 2016

\author{
Brandon Gilliland, Greg Macleod \\ Department of Neurology, Florida Atlantic University, USA
}

Correspondence: Dr. Gregory Macleod, Department of Neurology, Florida Atlantic University, USA,

Email macleodg@fau.edu

Received: May 25, 2016 | Published: December 30, 2016
Abbreviations: MS, multiple sclerosis; CSF, cerebro spinal fluid; RRMS, relapsing remitting multiple sclerosis; SPMS, secondary progressive multiple sclerosis; PPMS, primary-progressive multiple sclerosis; PRMS, progressive-relapsing multiple sclerosis; TNF, tumor necrosis factor; NMDA, N-methyl d-aspartate; SCI, spinal cord injury

\section{Introduction}

Multiple sclerosis (MS) is a disease in which multiple causes are being investigated. MS affects approximately 2.5 million people worldwide and about two hundred cases are diagnosed each week in the United States alone. ${ }^{1}$ The disease also affects women twice as much as men, and it is more prevalent in individuals of northern European descent. Ethnic groups at the lowest risk of developing MS are Native Americans, Africans, and Asians. "Interestingly, a child who relocates from an area of low risk to an area of high risk (or the other way around) takes on the risk level of the new location. However, if the child relocates are reaching puberty, he or she retains the risk level of the original location". ${ }^{1}$ This observation could suggest important environmental factors in MS. It is interesting to note that patients are often diagnosed with MS between the ages of 20 and 40 . With MS being the most widespread debilitating neurological disease of younger adults, it is important to understand its pathogenesis in efforts to find a cure.

Most patients diagnosed with MS experience symptoms such as distorted vision, lack of coordination and/or balance, unusual sensations, muscle weakness, bladder problems, etc. Three are four known classifications of MS: relapsing-remitting MS (RRMS), secondary-progressive MS (SPMS), primary-progressive MS (PPMS), and progressive-relapsing MS (PRMS). The most common form of MS is the relapsing-remitting, and it is recognized by exacerbations of worsening neurological function followed by remission periods. Approximately $85 \%$ of patients show this type of MS. ${ }^{2}$ Most patients who are diagnosed with RRMS eventually develop SPMS, a more steady form of the disease which involves fewer periods of remission. Approximately ten percent of patients with MS exhibit the primary-progressive (PPMS) form of the disease. This type of MS is characterized by conditions worsening in a linear progression with no distinct remission periods. The least common form of MS is progressive-relapsing (PRMS), and it involves a steady disease progression with exacerbations.

Multiple sclerosis is classified as a neurodegenerative autoimmune inflammatory disease. ${ }^{3}$ There have been debates over whether MS should be classified as an autoimmune disorder or simply a neurological disorder; however, most of the medical research indicates that the disease manifests the qualities of an autoimmune disorder. Oligodendrocytes are neuro glias that exist in the central nervous system to provide support to axons. These cells produce the myelin sheath that serves as insulation to the axon to enable salutatory action potentials. Oligodendrocytes cell death is seen in MS, and it is one of the main topics of interest in studying the disease's pathogenesis. Several theories have been developed to explain why oligodendrocytes are being destroyed in patients with MS.

\section{Theories of MS pathophysiology}

Many research groups have focused on the immune system itself in attempts to understand the pathogenesis of MS. The generally accepted view of oligodendrocytes cell death involves inflammation as a result of immune system activation. Acute MS lesions can be characterized as "active demyelination and inflammatory cell infiltrates, including $\mathrm{T}$ cells, B cells, macrophages, and activated microglia". ${ }^{4}$ A study published in 2001 by the Journal of Neuroscience Research showed the presence of myelin-reactive $T$ cells in the blood and cerebrospinal fluid (CSF) of MS patients. ${ }^{5}$ It has also been reported that "demyelination primarily results from a T-cell-mediated immune response to various myelin antigens. ${ }^{4}$ The theory adopted by Cadirci and others is that MS is associated with the up-regulation of TNF, TNF- $\beta$, IFN-g, and IL-12 pro-inflammatory cytokines. The production of these cytokines leads to the "up-regulation of endothelial cell adhesion molecules, to which $\mathrm{T}$ cells bind in a receptor-specific manner". 2 
This theory then suggests that the T cells enter into the perivascular space by way of the blood brain barrier. The $\mathrm{T}$ cells are able to enter into the blood brain barrier due to matrix proteases which degrade the extra-cellular matrix. It has also been noted that astrocytes and microglia can regulate inflammation by way of releasing cytokines, chemokine, and reactive oxygen species such as nitric oxide. ${ }^{2}$ Although there are many secondary processes involved in the theory of pathogenesis encompassing the up regulation of pro inflammatory cytokines, it is also important to consider the involvement of the tumor necrosis factor (TNF). Tumor Necrosis Factor (TNF), a mono-cytes derived cytotoxic, can promote both apoptotic and necrotic death of oligodendrocytes. The theory which involves the up regulation of pro inflammatory cytokines and the induction of oligodendrocytes cell death through TNFs seems to be a part of secondary processes rather than primary causes.

In order to understand some of the primary causes of MS which are not fully understood in science research currently, it is important to examine the effects of glutamate excitotoxicity and the environmental factors which could cause it. ${ }^{6}$ Glutamate excitotoxicity occurs when the AMPA/kainite type of glutamate receptors are over activated and cause damage to neurons and oligodendrocytes. Excitotoxicity is a pathological process which causes nerve cells to be damaged by excessive stimulation from neurotransmitters such as glutamate. Excitotoxins such as N-methyl-D-Aspartate (NMDA) and kainic acid bind to the NMDA receptor and excitotoxicity can occur through excessive glutamate stimulation through high levels of calcium entering into the cell. When the glutamate concentration around the synaptic cleft cannot be decreased, continued glutamate excitotoxicity will lead to apoptosis. The data suggest that this is what is causing the apoptosis in oligodendrocytes in MS patients. ${ }^{7}$

A spinal injury study published by Xu and colleagues in 2004 found that after spinal chord injury (SCI), damaged neural cells within the lesion site allow glutamate to enter into the extracellular space where glutamate can stimulate presynaptic glutamate receptors to drive the release of additional glutamate. This research study "demonstrate[d], in vivo that following SCI glutamate reaches concentrations toxic to white matter". When this occurs, glutamate toxicity ${ }^{8}$ to oligodendrocytes occurs via the AMPA/kainite receptors. ${ }^{9}$

N-methyl-D-Aspartate (NMDA) receptors are calcium favoring glutamate gated ion channels that are expressed in most neurons of the central nervous system. These receptors are important to consider because they are both ligand-gated and voltage-gated receptors. In order for activation to occur, the two different ligands, glutamate and either D-serine or glycine, must bind to the two activation sites. Continuous activation of large numbers of NMDA receptors (especially the NR1/NR2B-subtype) leads to increases in intracellular calcium loads and catabolic enzyme activities, which can trigger a cascade of events eventually leading to apoptosis or necrosis.

These downstream effects include mitochondrial membrane depolarization. ${ }^{10}$ Caspase activation, production of toxic oxygen and nitrogen free radicals, and cellular toxicity. ${ }^{11}$ Examination of these receptors is vital to a better understanding of the process of cell death, especially in neurodegenerative disease ${ }^{11}$ such as multiple sclerosis. In addition, mitochondria play an important role in mediating the state of the cell. "There is evidence that mitochondrial dysfunction and impairment of respiratory complexes play a role in the neuronal loss experienced in neurodegenerative Diseases. Recently, the mitochondrion has come to be considered a pivotal organelle in determining cell fate, because it may act as an 'on' switch modulating autophagy and apoptosis". ${ }^{11}$

\section{Conclusion}

Although there are different theories for the pathogenesis of MS, oligodendrocytes mediated cell death is one of the main problems with this neurodegenerative disease. Through extensive research in current medical literature, one can understand the different theories of the development and diagnosis of MS. With MS being the most widespread disabling neurological disease of young adults in the world today, it is important to continue to strive to find the primary cause of this neurodegenerative disease. There are many theories about the pathogenesis and progression of MS, including the result of a T-cell-mediated immune response to myelin antigens, glutamate excitotoxicity, mitochondrial dysfunction, and the result of environmental factors. The aspects of glutamate excitotoxicity and mitochondrial dysfunction are particular areas of interest that need to be considered more. Since there are many unknown factors currently regarding multiple sclerosis, there is a chance to discover the process and cure of a neurodegenerative disease that has affected many lives.

\section{Acknowledgements}

None.

\section{Conflict of interest}

The author declares no conflict of interest.

\section{References}

1. Pietrangelo A, Higuera V. Multiple Sclerosis by the Numbers: Facts, Statistics, and You. 2015.

2. http://www.nationalmssociety.org/What-is-MS/Types-of-MS

3. Brown G, Bal Price A. Inflammatory neurodegeneration mediated by nitric oxide, glutamate and mitochondria. Mol Neurobiol. 2003;27(3):325-355.

4. Cudrici C, Niculescu T, Niculescu F, et al. Oligodendrocyte cell death in pathogenesis of multiple sclerosis: Protection of oligodendrocytes from apoptosis by complement. J Rehabil Res Dev. 2006;43(1):123-132.

5. Hellings N, Baree M, Verhoeven C, et al. T-cell reactivity to multiple myelin antigens in multiple sclerosis patients and healthy controls. $J$ Neurosci Res. 2001;63(3):290-302.

6. Olney JW, Price MT, Samson L, et al. The role of specific ions in glutamate neurotoxicity. Neurosci Lett. 1986;28:65(1):65-71.

7. Yuan J, Yankner BA. Apoptosis in the nervous system. Nature. 2000;407(6805):802-809.

8. Maalouf M, Sullivan PG, Davis L, et al. Ketones inhibit mitochondrial production of reactive oxygen species production following glutamate excitotoxicity by increasing NADH oxidation. Neuroscience. 2007;145(1):256-264.

9. Xu G, Hughes M, Ye Z, et al. Concentrations of glutamate released following spinal cord injury kill oligodendrocytes in the spinal cord. Exp Neurol. 2004;187(2):329-336.

10. Duchen MR. Roles of mitochondria in health and disease. Diabetes. 2004;53 Supp11:S96-S102.

11. Dong XX, Wang Y, Qin ZH. Molecular mechanisms of excitotoxicity and their relevance to pathogenesis of neurodegenerative diseases. Acta Pharmacologica Sinica. 2009;30(4):379-387. 